ANNALES

POLONICI MATHEMATICI

$92.2(2007)$

\title{
On a boundary value problem on the half-line for nonlinear two-dimensional delay differential systems
}

\author{
by CH. G. Philos (Ioannina)
}

Dedicated to the memory of Professor M. K. Grammatikopoulos

\begin{abstract}
This article is concerned with a boundary value problem on the half-line for nonlinear two-dimensional delay differential systems. By the use of the SchauderTikhonov theorem, a result on the existence of solutions is obtained. Also, via the Banach contraction principle, another result concerning the existence and uniqueness of solutions is established. Moreover, these results are applied to the special case of ordinary differential systems and to a certain class of delay differential systems. Furthermore, applications to differential systems of Emden-Fowler type and to linear differential systems are presented, and two specific examples are given.
\end{abstract}

1. Introduction and statement of the main results. In the last few years, there is a great activity in studying the problem of the existence of solutions of boundary value problems on the half-line for second order nonlinear delay (and, in particular, ordinary) differential equations (see, for example, $[7,23,25,26,30,36,37,39,40])$. A closely related problem is that of the existence of solutions with prescribed asymptotic behavior for delay (and, especially, ordinary) differential equations. Among numerous articles dealing with this problem, we refer to [19, 20, 28, 29, 31, 32] and the references cited therein.

On the other hand, several articles have appeared in the literature, which are concerned with the asymptotic behavior of solutions of nonlinear ordinary differential systems. See, for example, [18, 21, 22, 38]; in particular, see the monograph by Mirzov [27] and the references therein.

For the basic theory of delay differential equations and systems, we refer to the books by Diekmann et al. [11], Driver [12], Hale [15], and Hale and

2000 Mathematics Subject Classification: 34K10, 34B40, 47H10.

Key words and phrases: delay differential system, boundary value problem on the half-line, existence of solutions, existence and uniqueness of solutions, Schauder-Tikhonov theorem, Banach contraction principle. 
Verduyn Lunel [16]. In particular, for infinite interval problems (including boundary value problems on the half-line), the reader is referred to the book by Agarwal and O'Regan [1].

This paper is essentially motivated by the recent work by Mavridis, the present author and Tsamatos [25] (the paper by the same authors [26] as well as the paper by Agarwal, the present author and Tsamatos [2] are closely related). Here, we deal with the existence of solutions and the existence and uniqueness of solutions of a boundary value problem on the half-line for nonlinear two-dimensional delay differential systems. Our results include, as special cases, those obtained in [25] for second order nonlinear delay differential equations.

For any interval $J$ of the real line and any subset $\Omega$ of $\mathbb{R}$, we denote by $C(J, \Omega)$ the set of all continuous functions defined on $J$ and having values in $\Omega$. Moreover, $r$ will be a nonnegative real number. Furthermore, if $t \in[0, \infty)$ and $\chi$ is a continuous real-valued function defined at least on $[t-r, t]$, the notation $\chi_{t}$ will be used for the function in $C([-r, 0], \mathbb{R})$ defined as follows:

$$
\chi_{t}(\tau)=\chi(t+\tau) \quad \text { for }-r \leq \tau \leq 0 .
$$

Notice that $C([-r, 0], \mathbb{R})$ is a Banach space with the sup-norm $\|\cdot\|$ :

$$
\|\omega\|=\max _{-r \leq \tau \leq 0}|\omega(\tau)| \quad \text { for } \omega \in C([-r, 0], \mathbb{R}) .
$$

Consider the nonlinear two-dimensional delay differential system

$$
x^{\prime}(t)=g(t, y(t)), \quad y^{\prime}(t)=-f\left(t, x_{t}, y(t)\right),
$$

where $g$ is a continuous real-valued function on $[0, \infty) \times \mathbb{R}$, and $f$ is a continuous real-valued function defined on $[0, \infty) \times C([-r, 0], \mathbb{R}) \times \mathbb{R}$.

Our interest concentrates on global solutions of (1.1), i.e., on solutions on the whole interval $[0, \infty)$. By a solution on $[0, \infty)$ of $(1.1)$, we mean two functions $x$ in $C([-r, \infty), \mathbb{R})$ and $y$ in $C([0, \infty), \mathbb{R})$, which are continuously differentiable on $[0, \infty)$ and satisfy (1.1) for all $t \geq 0$.

With (1.1), we associate an initial condition of the form

$$
x_{0}=\phi
$$

or, equivalently,

$$
x(t)=\phi(t) \quad \text { for }-r \leq t \leq 0,
$$

where $\phi$ in $C([-r, 0], \mathbb{R})$ is given. In what follows, it will be assumed that

$$
\phi(0)=0 \text {. }
$$

Also, along with (1.1), we impose a condition of the form

$$
\lim _{t \rightarrow \infty} y(t)=\xi
$$

where $\xi$ is a given real number. 
Equations (1.1)-(1.3) constitute a boundary value problem (BVP, for short) on the half-line.

A useful integral representation of the BVP (1.1)-(1.3) is given by Proposition 1.1 below, which will be used in proving the main results of the paper.

Proposition 1.1. Let $x \in C([-r, \infty), \mathbb{R})$ and $y \in C([0, \infty), \mathbb{R})$. Then $(x, y)$ is a solution of the BVP (1.1)-(1.3) if and only if

$$
x(t)= \begin{cases}\phi(t) & \text { for }-r \leq t \leq 0, \\ \int_{0}^{t} g(s, y(s)) d s & \text { for } t \geq 0\end{cases}
$$

and

$$
y(t)=\xi+\int_{t}^{\infty} f\left(s, x_{s}, y(s)\right) d s \quad \text { for } t \geq 0 .
$$

Proof. Suppose, first, that $(x, y)$ satisfies (1.4) and (1.5). Then we can easily see that $(x, y)$ is a solution of $(1.1)-(1.3)$.

Conversely, assume that $(x, y)$ is a solution of (1.1)-(1.3). In view of $\left(1.2^{\prime}\right)$, we have $x(0)=\phi(0)=0$. Hence, the first equation of $(1.1)$ gives

$$
x(t)=\int_{0}^{t} g(s, y(s)) d s \quad \text { for } t \geq 0,
$$

which together with $\left(1.2^{\prime}\right)$ guarantees that (1.4) is satisfied. Furthermore, from the second equation of (1.1) it follows that

$$
y(u)-y(t)=-\int_{t}^{u} f\left(s, x_{s}, y(s)\right) d s \quad \text { for } u \geq t \geq 0,
$$

and consequently, by (1.3),

$$
\xi-y(t)=-\int_{t}^{\infty} f\left(s, x_{s}, y(s)\right) d s \quad \text { for } t \geq 0,
$$

i.e., (1.5) holds true.

The proof is complete.

Our main results are Theorems 1.2 and 1.3 below. Theorem 1.2 provides sufficient conditions for the BVP (1.1)-(1.3) to have at least one solution, while Theorem 1.3 establishes sufficient conditions for the existence of a unique solution.

Theorem 1.2. Assume that

$$
|g(t, z)| \leq G(t,|z|) \quad \text { for all }(t, z) \in[0, \infty) \times \mathbb{R},
$$


where $G$ is a nonnegative continuous real-valued function on $[0, \infty) \times[0, \infty)$ which satisfies the following condition:

(A) For each $t \geq 0$, the function $G(t, \cdot)$ is increasing on $[0, \infty)$.

Moreover, assume that

$$
\begin{aligned}
& |f(t, \omega, z)| \leq F(t,|\omega|,|z|) \\
& \qquad \text { for all }(t, \omega, z) \in[0, \infty) \times C([-r, 0], \mathbb{R}) \times \mathbb{R},
\end{aligned}
$$

where $F$ is a nonnegative real-valued function defined on $[0, \infty) \times C([-r, 0]$, $[0, \infty)) \times[0, \infty)$, which satisfies the Continuity Condition: $F\left(t,\left|\chi_{t}\right|,|\psi(t)|\right)$ is continuous with respect to $t$ in $[0, \infty)$ for any given functions $\chi$ in $C([-r, \infty), \mathbb{R})$ and $\psi$ in $C([0, \infty), \mathbb{R})$. Suppose that:

(H) For each $t \geq 0$, the function $F(t, \cdot, \cdot)$ is increasing on $C([-r, 0],[0, \infty))$ $\times[0, \infty)$ in the sense that $F\left(t, \omega_{1}, z_{1}\right) \leq F\left(t, \omega_{2}, z_{2}\right)$ for any $\omega_{1}, \omega_{2}$ in $C([-r, 0],[0, \infty))$ with $\omega_{1} \leq \omega_{2}$ (i.e., $\omega_{1}(\tau) \leq \omega_{2}(\tau)$ for $\left.-r \leq \tau \leq 0\right)$ and any $z_{1}, z_{2}$ in $[0, \infty)$ with $z_{1} \leq z_{2}$.

Let there exist a real number $c$ with $c>|\xi|$ so that

$$
\int_{0}^{\infty} F\left(t, \eta_{t}, c\right) d t \leq c-|\xi|,
$$

where the function $\eta$ in $C([-r, \infty),[0, \infty))$ depends on $\phi, c, G$ and is defined by

$$
\eta(t)= \begin{cases}|\phi(t)| & \text { for }-r \leq t \leq 0, \\ \int_{0}^{t} G(s, c) d s & \text { for } t \geq 0 .\end{cases}
$$

Then the BVP (1.1)-(1.3) has at least one solution $(x, y)$ which satisfies

$$
-c+|\xi|+\xi \leq y(t) \leq c-|\xi|+\xi \quad \text { for every } t \geq 0
$$

if

$$
\text { for each } t \geq 0 \text {, the function } g(t, \cdot) \text { is increasing on } \mathbb{R},
$$

then it also satisfies

$$
\begin{aligned}
& \int_{0}^{t} g(s,-c+|\xi|+\xi) d s \\
& \quad \leq x(t) \leq \int_{0}^{t} g(s, c-|\xi|+\xi) d s \quad \text { for every } t \geq 0 .
\end{aligned}
$$

THEOREM 1.3. Let the assumptions of Theorem 1.2 hold. Furthermore, let the following generalized Lipschitz conditions be satisfied:

$$
\left|g\left(t, z_{1}\right)-g\left(t, z_{2}\right)\right| \leq L(t)\left|z_{1}-z_{2}\right| \text { for all }\left(t, z_{1}\right),\left(t, z_{2}\right) \text { in }[0, \infty) \times \mathbb{R}
$$


and

$$
\begin{aligned}
& \left|f\left(t, \omega_{1}, z_{1}\right)-f\left(t, \omega_{2}, z_{2}\right)\right| \leq K(t) \max \left\{\left\|\omega_{1}-\omega_{2}\right\|,\left|z_{1}-z_{2}\right|\right\} \\
& \qquad \text { for all }\left(t, \omega_{1}, z_{1}\right),\left(t, \omega_{2}, z_{2}\right) \text { in }[0, \infty) \times C([-r, 0], \mathbb{R}) \times \mathbb{R},
\end{aligned}
$$

where $L$ and $K$ are nonnegative continuous real-valued functions on the interval $[0, \infty)$ such that

$$
\int_{0}^{\infty} K(t) \max \left\{\int_{0}^{t} L(s) d s, 1\right\} d t<1 .
$$

Let there exist a real number $c$ with $c>|\xi|$ so that (1.8) holds, where $\eta$ is defined by (1.9). Then the BVP (1.1)-(1.3) has exactly one solution $(x, y)$ with

$$
|y(t)| \leq c \quad \text { for all } t \geq 0 \text {. }
$$

This unique solution satisfies (1.10); if condition (B) holds, it also satisfies (1.11).

2. Proofs of the theorems. To prove Theorem 1.2 we will use the Schauder-Tikhonov theorem (see [33, 34]).

The Schauder-Tikhonov Theorem. Let $\mu$ be a fixed positive continuous real-valued function on an interval $J$, and let $Y$ be the set of all functions $y$ in $C(J, \mathbb{R})$ which satisfy

$$
|y(t)| \leq \mu(t) \quad \text { for all } t \in J
$$

Let $M$ be a mapping of $Y$ into itself with the properties:

(i) $M$ is continuous in the sense that, for each $y$ in $Y$ and any sequence $\left(y_{n}\right)_{n \geq 1} \subset Y$, if $\lim _{n \rightarrow \infty} y_{n}=y$ uniformly on every compact subinterval of $J$, then $\lim _{n \rightarrow \infty} M y_{n}=M y$ uniformly on every such subinterval;

(ii) the image set $M Y$ is equicontinuous at every point of J.

Then $M$ has at least one fixed point in $Y$.

Let $V$ be a set of real-valued functions defined on an interval $J$, and let $t_{0} \in J$. The set $V$ is said to be equicontinuous at $t_{0}$ if, for each $\varepsilon>0$, there exists a $\delta \equiv \delta(\varepsilon)>0$ such that, for all $v$ in $V,\left|v(t)-v\left(t_{0}\right)\right|<\varepsilon$ for every $t \in J$ with $\left|t-t_{0}\right|<\delta$. Also, $V$ is called bounded at $t_{0}$ if there exists a $\Theta>0$ such that $\left|v\left(t_{0}\right)\right| \leq \Theta$ for all functions $v$ in $V$.

The Schauder-Tikhonov theorem has been stated, in the form presented above, by Coppel [8; p. 9], under the additional assumption that $M Y$ is bounded at every point of $J$. But this assumption is not needed. Indeed, as $M Y \subseteq Y$, it follows that $|(M y)(t)| \leq \mu(t)$ for all $y$ in $Y$ and every $t \in J$, which implies that $M Y$ is always bounded at every point of $J$. A proof of the 
Schauder-Tikhonov theorem stated above, based on Brouwer's well-known fixed point theorem for mappings in Euclidean spaces, can be found in [8; pp. 9-10].

In order to prove Theorem 1.3, we will make use of the Banach contraction principle (see Banach [3]; see also Kartsatos [17; p. 27]).

The Banach Contraction Principle. Let $E$ be a Banach space and $Y$ any nonempty closed subset of $E$. If $M$ is a contraction of $Y$ into itself, then $M$ has exactly one fixed point in $Y$.

Proof of Theorem 1.2. Let $Y$ be the set of all $y$ in $C([0, \infty), \mathbb{R})$ which satisfy $(1.15)$. For any $y$ in $Y$, let $x \in C([-r, \infty), \mathbb{R})$ be defined by $(1.4)$. (Note that $\phi(0)=0$.)

Fix $y$ in $Y$. Then, by (1.15) and assumption (A), we get

$$
G(t,|y(t)|) \leq G(t, c) \quad \text { for } t \geq 0 .
$$

But, in view of hypothesis (1.6),

$$
|g(t, y(t))| \leq G(t,|y(t)|) \quad \text { for } t \geq 0 .
$$

Hence, we have

$$
|g(t, y(t))| \leq G(t, c) \quad \text { for every } t \geq 0 .
$$

Thus, by taking into account the definition of $x$ by (1.4), we obtain, for $t \geq 0$,

$$
|x(t)| \leq\left|\int_{0}^{t} g(s, y(s)) d s\right| \leq \int_{0}^{t}|g(s, y(s))| d s \leq \int_{0}^{t} G(s, c) d s .
$$

So, by (1.9),

$$
|x(t)| \leq \eta(t) \quad \text { for every } t \geq-r
$$

and consequently

$$
\left|x_{t}\right| \leq \eta_{t} \quad \text { for all } t \geq 0 .
$$

Since $y \in Y$, using assumption $(\mathrm{H})$, we get

$$
F\left(t,\left|x_{t}\right|,|y(t)|\right) \leq F\left(t, \eta_{t}, c\right) \quad \text { for } t \geq 0 .
$$

On the other hand, hypothesis (1.7) gives

$$
\left|f\left(t, x_{t}, y(t)\right)\right| \leq F\left(t,\left|x_{t}\right|,|y(t)|\right) \quad \text { for } t \geq 0 .
$$

Consequently,

$$
\left|f\left(t, x_{t}, y(t)\right)\right| \leq F\left(t, \eta_{t}, c\right) \quad \text { for all } t \geq 0 .
$$

As hypothesis (1.8) implies, in particular, that

$$
\int_{0}^{\infty} F\left(t, \eta_{t}, c\right) d t<\infty
$$


by (2.1) we have

$$
\int_{0}^{\infty}\left|f\left(t, x_{t}, y(t)\right)\right| d t<\infty
$$

which guarantees that

$$
\int_{0}^{\infty} f\left(t, x_{t}, y(t)\right) d t \quad \text { exists in } \mathbb{R} .
$$

As (2.3) holds true for any $y \in Y$, the formula

$$
(M y)(t)=\xi+\int_{t}^{\infty} f\left(s, x_{s}, y(s)\right) d s \quad \text { for } t \geq 0
$$

makes sense for any $y$ in $Y$, and defines a mapping $M$ of $Y$ into $C([0, \infty), \mathbb{R})$. We first show that $M$ maps $Y$ into itself. Let $y \in Y$. Then, by using (2.1), from (2.4) we obtain, for $t \geq 0$,

$$
|(M y)(t)-\xi| \leq \int_{t}^{\infty}\left|f\left(s, x_{s}, y(s)\right)\right| d s \leq \int_{0}^{\infty}\left|f\left(s, x_{s}, y(s)\right)\right| d s \leq \int_{0}^{\infty} F\left(s, \eta_{s}, c\right) d s
$$

and consequently, in view of (1.8),

$$
|(M y)(t)-\xi| \leq c-|\xi| \quad \text { for all } t \geq 0,
$$

which obviously gives

$$
|(M y)(t)| \leq c \quad \text { for every } t \geq 0,
$$

so indeed $M y \in Y$.

Next, by (2.4) and (2.1), for any $y$ in $Y$ and all $t_{0}, t \geq 0$,

$$
\begin{aligned}
\left|(M y)(t)-(M y)\left(t_{0}\right)\right| & =\left|-\int_{t_{0}}^{t} f\left(s, x_{s}, y(s)\right) d s\right| \leq\left|\int_{t_{0}}^{t}\right| f\left(s, x_{s}, y(s)\right)|d s| \\
& \leq\left|\int_{t_{0}}^{t} F\left(s, \eta_{s}, c\right) d s\right| .
\end{aligned}
$$

So, by (2.2), MY is equicontinuous at every point $t_{0} \geq 0$.

Now, we prove that $M$ is continuous in the sense described in (i) of the Schauder-Tikhonov theorem. Let $y \in Y$ and $\left(y_{n}\right)_{n \geq 1} \subset Y$ with $\lim _{n \rightarrow \infty} y_{n}=y$ uniformly on every compact subinterval of $[0, \infty)$. For any integer $n \geq 1$, let $x_{n} \in C([-r, \infty), \mathbb{R})$ be defined by $(1.4)$ with $x_{n}$ in place of $x$ and $y_{n}$ instead of $y$, i.e.,

$$
x_{n}(t)= \begin{cases}\phi(t) & \text { for }-r \leq t \leq 0, \\ \int_{0}^{t} g\left(s, y_{n}(s)\right) d s & \text { for } t \geq 0 .\end{cases}
$$


By (2.1), we have

$$
\left|f\left(t,\left(x_{n}\right)_{t}, y_{n}(t)\right)\right| \leq F\left(t, \eta_{t}, c\right) \quad \text { for all } t \geq 0(n=1,2, \ldots) .
$$

So, because of (2.2), we can apply the Lebesgue dominated convergence theorem to obtain, for every $t \geq 0$,

$$
\lim _{n \rightarrow \infty} \int_{t}^{\infty} f\left(s,\left(x_{n}\right)_{s}, y_{n}(s)\right) d s=\int_{t}^{\infty} f\left(s, x_{s}, y(s)\right) d s .
$$

Hence $\lim _{n \rightarrow \infty} M y_{n}=M y$ pointwise on $[0, \infty)$. It remains to show that the convergence is uniform on every compact subinterval of $[0, \infty)$. To this end, let $\left(M y_{\lambda_{n}}\right)_{n \geq 1}$ be any subsequence of $\left(M y_{n}\right)_{n \geq 1}$. Since $M Y$ is equicontinuous at every point of $[0, \infty)$, and clearly also bounded at each point, the Arzelà-Ascoli theorem yields a subsequence $\left(M y_{\lambda_{\nu_{n}}}\right)_{n \geq 1}$ and a function $v$ in $C([0, \infty), \mathbb{R})$ such that $\lim _{n \rightarrow \infty} M y_{\lambda_{\nu_{n}}}=v$ uniformly on every compact subinterval of $[0, \infty)$. Since uniform convergence on every compact subinterval of $[0, \infty)$ implies pointwise convergence on $[0, \infty)$ to the same limit function, we have $v=M y$. So, $\left(M y_{n}\right)_{n \geq 1}$ converges to $M y$ uniformly on every compact subinterval of $[0, \infty)$. Consequently, $M$ is continuous.

We have seen that all assumptions of the Schauder-Tikhonov theorem are satisfied. Hence, there exists at least one $y$ in $Y$ with $y=M y$. By (2.4), $y$ satisfies (1.5). On the other hand, by the definition of $x,(1.4)$ is also satisfied. So, by Proposition $1.1,(x, y)$ is a solution of (1.1)-(1.3). As $y \in Y$ and $y=M y$, it follows from (2.5) that

$$
|y(t)-\xi| \leq c-|\xi| \quad \text { for every } t \geq 0
$$

and consequently (1.10) holds true. Finally, suppose that condition (B) is satisfied. Then, in view of (1.10),

$$
\begin{aligned}
\int_{0}^{t} g(s,-c+|\xi|+\xi) d s & \leq \int_{0}^{t} g(s, y(s)) d s \\
& \leq \int_{0}^{t} g(s, c-|\xi|+\xi) d s \quad \text { for every } t \geq 0
\end{aligned}
$$

which, because of (1.4), coincides with (1.11).

The proof of the theorem is complete.

Proof of Theorem 1.3. Consider the Banach space $E \equiv B C([0, \infty), \mathbb{R})$ of all bounded continuous real-valued functions on $[0, \infty)$, endowed with the sup-norm $\|\cdot\|$ defined by

$$
\|v\|=\sup _{t \geq 0}|v(t)| \quad \text { for } v \in B C([0, \infty), \mathbb{R}) .
$$

Consider also the set $Y$ of all $y$ in $C([0, \infty), \mathbb{R})$ which satisfy $(1.15)$. Clearly, $Y=\{y \in B C([0, \infty), \mathbb{R}):\|y\| \leq c\}$. The set $Y$ is a nonempty closed 
subset of $B C([0, \infty), \mathbb{R})$. For any $y$ in $Y$, we will denote by $x$ the function in $C([-r, \infty), \mathbb{R})$ defined by $(1.4)$. (We notice that $\phi(0)=0$.)

As in the proof of Theorem 1.2, we show that the formula (2.4) makes sense for any $y$ in $Y$, and defines a mapping $M$ of $Y$ into itself. We now prove that $M$ is a contraction. Let $y, \widetilde{y} \in Y$. Let $\widetilde{x} \in C([-r, \infty), \mathbb{R})$ be defined by (1.4) with $\widetilde{x}$ in place of $x$ and $\widetilde{y}$ in place of $y$, i.e.,

$$
\widetilde{x}(t)= \begin{cases}\phi(t) & \text { for }-r \leq t \leq 0, \\ \int_{0}^{t} g(s, \widetilde{y}(s)) d s & \text { for } t \geq 0\end{cases}
$$

By (1.13), for $t \geq 0$,

$$
\begin{aligned}
|(M y)(t)-(M \widetilde{y})(t)| & \leq \int_{t}^{\infty}\left|f\left(s, x_{s}, y(s)\right)-f\left(s, \widetilde{x}_{s}, \widetilde{y}(s)\right)\right| d s \\
& \leq \int_{t}^{\infty} K(s) \max \left\{\left\|x_{s}-\widetilde{x}_{s}\right\|,|y(s)-\widetilde{y}(s)|\right\} d s .
\end{aligned}
$$

Consequently,

$$
\begin{aligned}
\|M y-M \widetilde{y}\| & =\sup _{t \geq 0}|(M y)(t)-(M \widetilde{y})(t)| \\
& \leq \int_{0}^{\infty} K(t) \max \left\{\left\|x_{t}-\widetilde{x}_{t}\right\|,|y(t)-\widetilde{y}(t)|\right\} d t .
\end{aligned}
$$

By (1.4) and (2.6), we have $x(t)=\widetilde{x}(t)=\phi(t)$ for $-r \leq t \leq 0$ and so

$$
|x(t)-\widetilde{x}(t)|=0 \quad \text { for }-r \leq t \leq 0 .
$$

Furthermore, by (1.4) and (2.6) as well as the hypothesis (1.12), we obtain, for $t \geq 0$,

$$
|x(t)-\widetilde{x}(t)| \leq \int_{0}^{t}|g(s, y(s))-g(s, \widetilde{y}(s))| d s \leq \int_{0}^{t} L(s)|y(s)-\widetilde{y}(s)| d s .
$$

But it is obvious that

$$
|y(t)-\widetilde{y}(t)| \leq\|y-\widetilde{y}\| \quad \text { for all } t \geq 0 .
$$

Thus, we get

$$
|x(t)-\widetilde{x}(t)| \leq\left[\int_{0}^{t} L(s) d s\right]\|y-\widetilde{y}\| \quad \text { for every } t \geq 0 .
$$

This inequality together with (2.8) implies that

$$
|x(t)-\widetilde{x}(t)| \leq \sigma(t)\|y-\widetilde{y}\| \quad \text { for all } t \geq-r,
$$


where

$$
\sigma(t)=0 \quad \text { for }-r \leq t \leq 0, \quad \sigma(t)=\int_{0}^{t} L(s) d s \quad \text { for } t \geq 0 .
$$

Therefore,

$$
|x(t+\tau)-\widetilde{x}(t+\tau)| \leq \sigma(t+\tau)\|y-\widetilde{y}\| \quad \text { for } t \geq 0 \text { and }-r \leq \tau \leq 0,
$$
i.e.,

$$
\left|x_{t}(\tau)-\widetilde{x}_{t}(\tau)\right| \leq \sigma(t+\tau)\|y-\widetilde{y}\| \quad \text { for } t \geq 0 \text { and }-r \leq \tau \leq 0 .
$$

This gives

$$
\max _{-r \leq \tau \leq 0}\left|x_{t}(\tau)-\widetilde{x}_{t}(\tau)\right| \leq\left[\max _{-r \leq \tau \leq 0} \sigma(t+\tau)\right]\|y-\widetilde{y}\| \quad \text { for } t \geq 0 .
$$

But, as $\sigma$ is increasing on $[-r, \infty)$, we have

$$
\max _{-r \leq \tau \leq 0} \sigma(t+\tau)=\sigma(t)=\int_{0}^{t} L(s) d s \quad \text { for } t \geq 0 .
$$

Hence,

$$
\left\|x_{t}-\widetilde{x_{t}}\right\| \leq\left[\int_{0}^{t} L(s) d s\right]\|y-\widetilde{y}\| \quad \text { for all } t \geq 0 .
$$

By using this inequality as well as (2.9), from (2.7) we obtain

$$
\|M y-M \widetilde{y}\| \leq\left[\int_{0}^{\infty} K(t) \max \left\{\int_{0}^{t} L(s) d s, 1\right\} d t\right]\|y-\widetilde{y}\| .
$$

Thus, by hypothesis (1.14), $M$ is a contraction, so it has a unique fixed point $y$ in $Y$. By Proposition 1.1, this means that the BVP (1.1)-(1.3) has exactly one solution $(x, y)$ satisfying (1.15). As in the proof of Theorem 1.2, this unique solution is such that (1.10) holds; in addition, when condition (B) is satisfied, (1.11) also holds.

The proof of the theorem is now complete.

\section{Application to ordinary differential systems and to a specific} class of delay differential systems. Consider, first, the nonlinear twodimensional ordinary differential system

$$
x^{\prime}(t)=g(t, y(t)), \quad y^{\prime}(t)=-f_{0}(t, x(t), y(t)),
$$

where $f_{0}$ is a continuous real-valued function on $[0, \infty) \times \mathbb{R}^{2}$.

We restrict our attention to solutions of $(3.1)$ on the whole interval $[0, \infty)$. With (3.1), we associate the initial condition

$$
x(0)=0
$$


as well as condition (1.3). In this special case, the BVP (1.1)-(1.3) reduces to the BVP (3.1), (3.2), (1.3).

By specifying Theorems 1.2 and 1.3 to this case, we get Corollaries 3.1 and 3.2 below.

Corollary 3.1. Assume that (1.6) holds, where $G$ is a nonnegative continuous real-valued function on $[0, \infty) \times[0, \infty)$ which satisfies condition $(\mathrm{A})$. Moreover, assume that

$$
\left|f_{0}(t, w, z)\right| \leq F_{0}(t,|w|,|z|) \quad \text { for all }(t, w, z) \in[0, \infty) \times \mathbb{R}^{2},
$$

where $F_{0}$ is a nonnegative continuous real-valued function on $[0, \infty) \times[0, \infty)^{2}$. Suppose that, for each $t \geq 0$, the function $F_{0}(t, \cdot, \cdot)$ is increasing on $[0, \infty)^{2}$. Let there exist a real number $c$ with $c>|\xi|$ so that

$$
\int_{0}^{\infty} F_{0}\left(t, \int_{0}^{t} G(s, c) d s, c\right) d t \leq c-|\xi| .
$$

Then the BVP (3.1), (3.2), (1.3) has at least one solution $(x, y)$ which satisfies (1.10); if condition (B) holds, it also satisfies (1.11).

Corollary 3.2. Let the assumptions of Corollary 3.1 hold. Furthermore, let the generalized Lipschitz conditions (1.12) and

$$
\begin{aligned}
\left|f_{0}\left(t, w_{1}, z_{1}\right)-f_{0}\left(t, w_{2}, z_{2}\right)\right| \leq & K(t) \max \left\{\left|w_{1}-w_{2}\right|,\left|z_{1}-z_{2}\right|\right\} \\
& \quad \text { for all }\left(t, w_{1}, z_{1}\right),\left(t, w_{2}, z_{2}\right) \text { in }[0, \infty) \times \mathbb{R}^{2}
\end{aligned}
$$

be satisfied, where $L$ and $K$ are nonnegative continuous real-valued functions on the interval $[0, \infty)$ such that $(1.14)$ holds. Let there exist a real number $c$ with $c>|\xi|$ so that (3.3) holds. Then the BVP (3.1), (3.2), (1.3) has exactly one solution $(x, y)$ satisfying (1.15). This unique solution satisfies $(1.10)$; if condition (B) holds, it also satisfies (1.11).

Next, consider the nonlinear two-dimensional delay differential system

$$
\left\{\begin{array}{l}
x^{\prime}(t)=g(t, y(t)) \\
y^{\prime}(t)=-f_{1}\left(t, x\left(t-T_{1}(t)\right), \ldots, x\left(t-T_{m}(t)\right), y(t)\right),
\end{array}\right.
$$

where $m$ is a positive integer, $f_{1}$ is a continuous real-valued function on $[0, \infty) \times \mathbb{R}^{m+1}$, and $T_{j}(j=1, \ldots, m)$ are nonnegative continuous real-valued functions on $[0, \infty)$ with

$$
\max _{j=1, \ldots, m} \sup _{t \geq 0} T_{j}(t)=r .
$$

A solution on $[0, \infty)$ of $(3.4)$ is two functions $x$ in $C([-r, \infty), \mathbb{R})$ and $y$ in $C([0, \infty), \mathbb{R})$, which are continuously differentiable on $[0, \infty)$ and satisfy (3.4) for all $t \geq 0$. The initial condition $\left(1.2^{\prime}\right)$ as well as condition (1.3) are associated with (3.4). In this case, the BVP (1.1)-(1.3) reduces to the BVP $(3.4),\left(1.2^{\prime}\right),(1.3)$. 
If the delay differential system (1.1) is to be equivalent to (3.4), we must define

$$
f(t, \omega, z)=f_{1}\left(t, \omega\left(-T_{1}(t)\right), \ldots, \omega\left(-T_{m}(t)\right), z\right)
$$

for $(t, \omega, z) \in[0, \infty) \times C([-r, 0], \mathbb{R}) \times \mathbb{R}$. Hence, by applying Theorems 1.2 and 1.3 to the BVP $(3.4),\left(1.2^{\prime}\right),(1.3)$, we are led to Corollaries 3.3 and 3.4 below.

Corollary 3.3. Assume that (1.6) holds, where $G$ is a nonnegative continuous real-valued function on $[0, \infty) \times[0, \infty)$, which satisfies condition $(\mathrm{A})$. Moreover, assume that

$$
\begin{aligned}
& \left|f_{1}\left(t, w_{1}, \ldots, w_{m}, z\right)\right| \leq F_{1}\left(t,\left|w_{1}\right|, \ldots,\left|w_{m}\right|,|z|\right) \\
& \qquad \text { for all }\left(t, w_{1}, \ldots, w_{m}, z\right) \in[0, \infty) \times \mathbb{R}^{m+1},
\end{aligned}
$$

where $F_{1}$ is a nonnegative continuous real-valued function on $[0, \infty) \times$ $[0, \infty)^{m+1}$. Suppose that, for each $t \geq 0$, the function $F_{1}(t, \cdot, \ldots, \cdot, \cdot)$ is increasing on $[0, \infty)^{m+1}$. Let there exist a real number $c$ with $c>|\xi|$ so that

$$
\int_{0}^{\infty} F_{1}\left(t, \varrho_{1}(t), \ldots, \varrho_{m}(t), c\right) d t \leq c-|\xi|,
$$

where, for each $j \in\{1, \ldots, m\}$, the function $\varrho_{j}$ in $C([0, \infty),[0, \infty))$ depends on $\phi, c, G$ and is defined by

$$
\varrho_{j}(t)= \begin{cases}\left|\phi\left(t-T_{j}(t)\right)\right| & \text { if } 0 \leq t \leq T_{j}(t), \\ \int_{0}^{t-T_{j}(t)} G(s, c) d s & \text { if } t \geq T_{j}(t) .\end{cases}
$$

Then the BVP (3.4), (1.2'), (1.3) has at least one solution $(x, y)$ which satisfies (1.10); if condition (B) holds, it also satisfies (1.11).

Corollary 3.4. Let the assumptions of Corollary 3.3 hold. Furthermore, let the generalized Lipschitz conditions (1.12) and

$$
\begin{aligned}
\left|f_{1}\left(t, w_{1}^{(1)}, \ldots, w_{m}^{(1)}, z_{1}\right)-f_{1}\left(t, w_{1}^{(2)}, \ldots, w_{m}^{(2)}, z_{2}\right)\right| \\
\quad \leq K(t) \max \left\{\left|w_{1}^{(1)}-w_{1}^{(2)}\right|, \ldots,\left|w_{m}^{(1)}-w_{m}^{(2)}\right|,\left|z_{1}-z_{2}\right|\right\}
\end{aligned}
$$

for all $\left(t, w_{1}^{(1)}, \ldots, w_{m}^{(1)}, z_{1}\right),\left(t, w_{1}^{(2)}, \ldots, w_{m}^{(2)}, z_{2}\right)$ in $[0, \infty) \times \mathbb{R}^{m+1}$ be satisfied, where $L$ and $K$ are nonnegative continuous real-valued functions on $[0, \infty)$ such that $(1.14)$ holds. Let there exist a real number $c$ with $c>|\xi|$ so that (3.5) holds, where, for each $j \in\{1, \ldots, m\}, \varrho_{j}$ is defined by (3.6). Then the BVP (3.4), (1.2'), (1.3) has exactly one solution $(x, y)$ satisfying (1.15). This unique solution satisfies (1.10); if condition (B) holds, it also satisfies (1.11). 
4. Application to differential systems of Emden-Fowler type and to linear differential systems. Examples. Consider the nonlinear two-dimensional Emden-Fowler ordinary differential system

$$
\left\{\begin{array}{l}
x^{\prime}(t)=q(t)|y(t)|^{\sigma} \operatorname{sgn} y(t), \\
y^{\prime}(t)=-a(t)|x(t)|^{\gamma} \operatorname{sgn} x(t)-b(t)|y(t)|^{\beta} \operatorname{sgn} y(t),
\end{array}\right.
$$

as well as the nonlinear two-dimensional delay differential system of EmdenFowler type

$$
\left\{\begin{array}{l}
x^{\prime}(t)=q(t)|y(t)|^{\sigma} \operatorname{sgn} y(t), \\
y^{\prime}(t)=-a(t)|x(t-r)|^{\gamma} \operatorname{sgn} x(t-r)-b(t)|y(t)|^{\beta} \operatorname{sgn} y(t),
\end{array}\right.
$$

where $q, a$ and $b$ are continuous real-valued functions on the interval $[0, \infty)$, and $\sigma, \gamma$ and $\beta$ are positive real numbers. Consider also the linear twodimensional ordinary differential system

$$
x^{\prime}(t)=q(t) y(t), \quad y^{\prime}(t)=-a(t) x(t)-b(t) y(t)
$$

as well as the linear two-dimensional delay differential system

$$
x^{\prime}(t)=q(t) y(t), \quad y^{\prime}(t)=-a(t) x(t-r)-b(t) y(t) .
$$

We denote by $Q$ the nonnegative continuous real-valued function on $[0, \infty)$ defined by the formula

$$
Q(t)=\int_{0}^{t}|q(s)| d s \quad \text { for } t \geq 0 .
$$

By applying Theorem 1.2 (more specifically, Corollary 3.1) to the BVP (4.1), (3.2), (1.3), we are led to Corollary 4.1 below. Similarly, in the case of the BVP (4.2), (1.2'), (1.3), Theorem 1.2 (more specifically, Corollary 3.3) leads to Corollary 4.2 below.

COROLlary 4.1. Let there exist a real number $c$ with $c>|\xi|$ so that

$$
c^{\sigma \gamma} \int_{0}^{\infty}[Q(t)]^{\gamma}|a(t)| d t+c^{\beta} \int_{0}^{\infty}|b(t)| d t \leq c-|\xi| .
$$

Then the BVP (4.1), (3.2), (1.3) has at least one solution $(x, y)$ which satisfies (1.10); if

$$
q \text { is nonnegative on }[0, \infty) \text {, }
$$

it also satisfies

$$
\begin{aligned}
& {\left[|-c+| \xi|+\xi|^{\sigma} \operatorname{sgn}(-c+|\xi|+\xi)\right] \int_{0}^{t} q(s) d s \leq x(t)} \\
& \leq\left[|c-| \xi|+\xi|^{\sigma} \operatorname{sgn}(c-|\xi|+\xi)\right] \int_{0}^{t} q(s) d s \quad \text { for every } t \geq 0 .
\end{aligned}
$$


COROllary 4.2. Let there exist a real number $c$ with $c>|\xi|$ so that

$$
\begin{aligned}
& \int_{0}^{r}|\phi(t-r)|^{\gamma}|a(t)| d t+c^{\sigma \gamma} \int_{r}^{\infty}[Q(t-r)]^{\gamma}|a(t)| d t \\
& +c^{\beta} \int_{0}^{\infty}|b(t)| d t \leq c-|\xi| .
\end{aligned}
$$

Then the BVP (4.2), $\left(1.2^{\prime}\right),(1.3)$ has at least one solution $(x, y)$ which satisfies (1.10); if (4.6) holds, it also satisfies (4.7).

If we apply Theorem 1.3 (more specifically, Corollary 3.2) to the BVP (4.3), (3.2), (1.3), then we arrive at Corollary 4.3 below. Moreover, by an application of Theorem 1.3 (more specifically, of Corollary 3.4) to the BVP (4.4), (1.2'), (1.3), Corollary 4.4 below is obtained.

Corollary 4.3. Assume that

$$
\int_{0}^{\infty} \max \{Q(t), 1\}[|a(t)|+|b(t)|] d t<1 .
$$

Let there exist a real number $c$ with $c>|\xi|$ so that

$$
c\left[\int_{0}^{\infty} Q(t)|a(t)| d t+\int_{0}^{\infty}|b(t)| d t\right] \leq c-|\xi| .
$$

Then the BVP (4.3), (3.2), (1.3) has exactly one solution $(x, y)$ satisfying (1.15). This unique solution satisfies (1.10); if (4.6) holds, it also satisfies

$$
\begin{aligned}
(-c+|\xi|+\xi) \int_{0}^{t} q(s) d s & \leq x(t) \\
& \leq(c-|\xi|+\xi) \int_{0}^{t} q(s) d s \quad \text { for every } t \geq 0 .
\end{aligned}
$$

Corollary 4.4. Assume that condition (4.9) is satisfied. Let there exist a real number $c$ with $c>|\xi|$ so that

$$
\int_{0}^{r}|\phi(t-r)||a(t)| d t+c\left[\int_{r}^{\infty} Q(t-r)|a(t)| d t+\int_{0}^{\infty}|b(t)| d t\right] \leq c-|\xi| .
$$

Then the BVP (4.4), (1.2'), (1.3) has exactly one solution $(x, y)$ satisfying (1.15). This unique solution satisfies (1.10); if (4.6) holds, it also satisfies (4.11).

Note that, by taking $r=0$, systems (4.2) and (4.4) lead to (4.1) and (4.3) respectively. On the other hand, conditions (4.5) and (4.10) are obtained from (4.8) and (4.12), respectively, for $r=0$. 
Assume that condition (4.9) is satisfied. Since

$$
\int_{0}^{\infty} Q(t)|a(t)| d t+\int_{0}^{\infty}|b(t)| d t \leq \int_{0}^{\infty} \max \{Q(t), 1\}[|a(t)|+|b(t)|] d t,
$$

it follows from (4.9) that

$$
\int_{0}^{\infty} Q(t)|a(t)| d t+\int_{0}^{\infty}|b(t)| d t<1 .
$$

Furthermore, we see that assumption (4.10) is equivalently written as

$$
c\left[1-\int_{0}^{\infty} Q(t)|a(t)| d t-\int_{0}^{\infty}|b(t)| d t\right] \geq|\xi| .
$$

Suppose, first, that $\xi=0$. Then, by (4.13), we immediately see that (4.14) holds true for any $c>0=|\xi|$. Next, suppose that $\xi \neq 0$. Assume that at least one of the functions $Q a$ and $b$ is not identically zero on $[0, \infty)$. This assumption guarantees that

$$
\int_{0}^{\infty} Q(t)|a(t)| d t+\int_{0}^{\infty}|b(t)| d t>0 .
$$

By (4.13) and (4.15), the formula

$$
c=\frac{|\xi|}{1-\int_{0}^{\infty} Q(t)|a(t)| d t-\int_{0}^{\infty}|b(t)| d t}
$$

defines a real number $c$ with $c>|\xi|$. For this real number $c$, inequality (4.14) holds true (as an equality). After the above observations, we conclude that Corollary 4.3 leads to the following result.

Corollary 4.5. Assume that condition (4.9) is satisfied. Then we have:

(i) Let $c$ be any positive real number. Then the BVP (4.3), (3.2),

$$
\lim _{t \rightarrow \infty} y(t)=0
$$

has exactly one solution ( $x, y)$ satisfying (1.15); if (4.6) holds, it also satisfies

$$
-c \int_{0}^{t} q(s) d s \leq x(t) \leq c \int_{0}^{t} q(s) d s \quad \text { for every } t \geq 0 .
$$

(ii) Suppose that $Q a$ or $b$ is not identically zero on $[0, \infty)$. Let $\xi \neq 0$ and let $c$ be defined by (4.16). ( $c$ is a real number with $c>|\xi|)$. Then the conclusion of Corollary 4.3 holds.

By an analysis similar to that in deducing Corollary 4.5 from Corollary 4.3, one can derive Corollary 4.6 below from Corollary 4.4. We restrict 
ourselves to noting that

$$
\begin{aligned}
\int_{r}^{\infty} Q(t-r)|a(t)| d t+\int_{0}^{\infty}|b(t)| d t & \leq \int_{r}^{\infty} Q(t)|a(t)| d t+\int_{0}^{\infty}|b(t)| d t \\
& \leq \int_{0}^{\infty} Q(t)|a(t)| d t+\int_{0}^{\infty}|b(t)| d t \\
& \leq \int_{0}^{\infty} \max \{Q(t), 1\}[|a(t)|+|b(t)|] d t
\end{aligned}
$$

and so condition (4.9) implies that

$$
\int_{r}^{\infty} Q(t-r)|a(t)| d t+\int_{0}^{\infty}|b(t)| d t<1 .
$$

Corollary 4.6. Assume that condition (4.9) is satisfied. Then we have:

(i) Let c be any positive real number. Then the BVP (4.4), (4.19), (4.17), where

$$
x(t)=0 \quad \text { for }-r \leq t \leq 0,
$$

has exactly one solution $(x, y)$ satisfying (1.15); if (4.6) holds, it also satisfies (4.18).

(ii) Define $U(t)=Q(t-r) a(t)$ for $t \geq r$. Suppose that $U$ is not identically zero on $[r, \infty)$ or $b$ is not identically zero on $[0, \infty)$. Let $\xi \neq 0$ and let $c$ be defined by

$$
c=\frac{|\xi|+\int_{0}^{r}|\phi(t-r)||a(t)| d t}{1-\int_{r}^{\infty} Q(t-r)|a(t)| d t-\int_{0}^{\infty}|b(t)| d t}
$$

( $c$ is a real number with $c>|\xi|$ ). Then the conclusion of Corollary 4.4 holds.

Now, in order to demonstrate the applicability of our results, we give two specific examples. We restrict ourselves to ordinary differential systems. In Example 4.7, Corollary 4.1 is applicable, while in Example 4.8, Corollary 4.5 can be used.

ExAmple 4.7. Consider the nonlinear two-dimensional Emden-Fowler ordinary differential system

$$
\left\{\begin{array}{l}
x^{\prime}(t)=\frac{2 t}{(t+1)^{3}}[y(t)]^{4} \operatorname{sgn} y(t), \\
y^{\prime}(t)=-\frac{t}{3(t+1)^{3}}|x(t)|^{1 / 2} \operatorname{sgn} x(t)-\frac{1}{6(t+1)^{2}} y(t),
\end{array}\right.
$$

with the initial condition (3.2) as well as the condition

$$
\lim _{t \rightarrow \infty} y(t)=1 \text {. }
$$


For the BVP (4.20), (3.2), (4.21), condition (4.5) becomes

$$
c^{2} \int_{0}^{\infty} \frac{t^{2}}{3(t+1)^{4}} d t+c \int_{0}^{\infty} \frac{d t}{6(t+1)^{2}} \leq c-1 .
$$

This inequality can easily be written as

$$
\frac{1}{9} c^{2}+\frac{1}{6} c \leq c-1, \quad \text { i.e., } \quad\left(c-\frac{3}{2}\right)(c-6) \leq 0 .
$$

So, (4.5) is satisfied if and only if $3 / 2 \leq c \leq 6$. Take $c=3 / 2$. By Corollary 4.1, the BVP (4.20), (3.2), (4.21) has at least one solution $(x, y)$ which satisfies

$$
1 / 2 \leq y(t) \leq 3 / 2 \quad \text { for every } t \geq 0
$$

and

$$
\frac{t^{2}}{16(t+1)^{2}} \leq x(t) \leq \frac{81 t^{2}}{16(t+1)^{2}} \quad \text { for every } t \geq 0 .
$$

EXAMPLE 4.8. Consider the linear two-dimensional ordinary differential system

$$
x^{\prime}(t)=\frac{2 t}{(t+1)^{3}} y(t), \quad y^{\prime}(t)=-\frac{1}{2(t+1)^{2}} x(t)-\frac{1}{6(t+1)^{2}} y(t),
$$

with the initial condition (3.2) as well as the condition (4.21). Here, condition (4.9) becomes

$$
\int_{0}^{\infty} \max \left\{\frac{t^{2}}{(t+1)^{2}}, 1\right\}\left[\frac{1}{2(t+1)^{2}}+\frac{1}{6(t+1)^{2}}\right] d t<1 .
$$

It is easy to verify that this inequality holds true. Furthermore, in this case, (4.16) reads

$$
c=\frac{1}{1-\int_{0}^{\infty} \frac{t^{2}}{2(t+1)^{4}} d t-\int_{0}^{\infty} \frac{d t}{6(t+1)^{2}}}
$$

and so $c=3 / 2$. By Corollary 4.5, the BVP (4.22), (3.2), (4.21) has exactly one solution $(x, y)$ with

$$
|y(t)| \leq 3 / 2 \text { for all } t \geq 0
$$

this unique solution $(x, y)$ satisfies

$$
1 / 2 \leq y(t) \leq 3 / 2 \text { for every } t \geq 0
$$

and

$$
\frac{t^{2}}{2(t+1)^{2}} \leq x(t) \leq \frac{3 t^{2}}{2(t+1)^{2}} \quad \text { for every } t \geq 0
$$


5. Discussion. It is an open problem whether the assertions of Theorem 1.3 and Corollaries 3.2 and 3.4 remain valid with the weaker assumption

$$
\int_{0}^{\infty} K(t) \max \left\{\int_{0}^{t} L(s) d s, 1\right\} d t<\infty
$$

in place of (1.14). In particular, it is an open question if hypothesis (4.9) in Corollaries $4.3-4.6$ can be replaced by the weaker condition

$$
\int_{0}^{\infty} \max \{Q(t), 1\}[|a(t)|+|b(t)|] d t<\infty .
$$

To investigate the above open problems, one must use a renormalization procedure due to Bielecki [6]; this technique is very useful in obtaining global existence criteria and in studying several stability problems (see, for example, the books $[9,10]$ and the recent papers $[13,14,35])$.

Let us consider the second order nonlinear delay differential equation

$$
\left[k(t) x^{\prime}(t)\right]^{\prime}+f\left(t, x_{t}, k(t) x^{\prime}(t)\right)=0,
$$

where $k$ is a positive continuous real-valued function on $[0, \infty)$. With (5.1), we associate the initial condition (1.2) as well as the condition

$$
\lim _{t \rightarrow \infty} k(t) x^{\prime}(t)=\xi \text {. }
$$

The substitution $k x^{\prime}=y$ transforms (5.1) into the equivalent nonlinear two-dimensional delay differential system

$$
x^{\prime}(t)=\frac{1}{k(t)} y(t), \quad y^{\prime}(t)=-f\left(t, x_{t}, y(t)\right) .
$$

By applying Theorems 1.2 and 1.3 to the BVP (5.3), (1.2), (1.3), we obtain two results concerning the BVP (5.1), (1.2), (5.2). In particular, in the special case where $k(t)=1$ for $t \geq 0$, the application of Theorem 1.2 to the BVP (5.1), (1.2), (1.3) leads to the main result in the work by Mavridis, the present author and Tsamatos [25] which concerns the BVP (5.1), (1.2), (5.2) (with $k \equiv 1)$.

Now, consider the nonlinear two-dimensional delay differential system

$$
x^{\prime}(t)=d(t, y(t)), \quad y^{\prime}(t)=-h\left(t, x_{t}, y(t)\right),
$$

where $d$ is a continuous real-valued function on $(0, \infty) \times \mathbb{R}$, and $h$ is a continuous real-valued function on $(0, \infty) \times C([-r, 0], \mathbb{R}) \times \mathbb{R}$. We are interested in global solutions of (5.4); such a solution is a pair of functions $x$ in $C([-r, \infty), \mathbb{R})$ and $y$ in $C((0, \infty), \mathbb{R})$, which are continuously differentiable on $(0, \infty)$ and satisfy $(5.4)$ for all $t>0$. With (5.4), one associates the initial condition (1.2) as well as another initial condition of the form

$$
\lim _{t \rightarrow 0+} y(t)=\theta
$$


where $\theta$ is a given real number. Equations (5.4), (1.2), (5.5) constitute an initial value problem (IVP, for short).

Next, consider the second order nonlinear delay differential equation

$$
\left[p(t) x^{\prime}(t)\right]^{\prime}+h\left(t, x_{t}, p(t) x^{\prime}(t)\right)=0,
$$

where $p$ is a positive continuous real-valued function on $(0, \infty)$. With (5.6), we associate the initial condition (1.2) and the initial condition

$$
\lim _{t \rightarrow 0+} p(t) x^{\prime}(t)=\theta .
$$

By setting $y=p x^{\prime}$, the delay differential equation (5.6) is transformed into the equivalent nonlinear two-dimensional delay differential system

$$
x^{\prime}(t)=\frac{1}{p(t)} y(t), \quad y^{\prime}(t)=-h\left(t, x_{t}, y(t)\right) .
$$

That is, the substitution $y=p x^{\prime}$ transforms the IVP (5.6), (1.2), (5.7) into the equivalent IVP (5.8), (1.2), (5.5).

Applying the Schauder theorem and the Banach contraction principle, Agarwal, the author and Tsamatos [2] studied the existence of global solutions as well as the existence and uniqueness of global solutions to the singular IVP (5.6), (1.2), (5.7). For some previous related results, see $[4,5,24]$. By a combination of the methods applied in [2] with the ideas and techniques of this paper, one can extend the results in [2] to the more general case of the singular IVP (5.4), (1.2), (5.5).

\section{References}

[1] R. P. Agarwal and D. O'Regan, Infinite Interval Problems for Differential, Difference and Integral Equations, Kluwer, Dordrecht, 2001.

[2] R. P. Agarwal, Ch. G. Philos and P. Ch. Tsamatos, Global solutions of a singular initial value problem to second order nonlinear delay differential equations, Math. Comput. Modelling 43 (2006), 854-869.

[3] S. Banach, Sur les opérations dans les ensembles abstraits et leur application aux équations intégrales, Fund. Math. 3 (1922), 133-181.

[4] J. V. Baxley, Existence and uniqueness for nonlinear boundary value problems on infinite intervals, J. Math. Anal. Appl. 147 (1990), 122-133.

[5] J. V. Baxley and C. G. Enloe, Nonlinear initial-value problems with positive global solutions, Electron. J. Differ. Equ. Conf. 10 (2003), 71-78.

[6] A. Bielecki, Une remarque sur la méthode de Banach-Caccioppoli-Tikhonov dans la théorie des équations différentielles ordinaires, Bull. Acad. Polon. Sci. Cl. III 4 (1956), 261-264.

[7] A. Constantin, On the existence of positive solutions of second order differential equations, Ann. Mat. Pura Appl. 184 (2005), 131-138.

[8] W. A. Coppel, Stability and Asymptotic Behavior of Differential Equations, Heath, Boston, 1965.

[9] C. Corduneanu, Integral Equations and Stability of Feedback Systems, Math. Sci. Engrg. 104, Academic Press, New York, 1973. 
[10] C. Corduneanu, Integral Equations and Applications, Cambridge Univ. Press, Cambridge, 1991.

[11] O. Diekmann, S. A. van Gils, S. M. Verduyn Lunel and H.-O. Walther, Delay Equations: Functional, Complex, and Nonlinear Analysis, Appl. Math. Sci. 110, Springer, New York, 1995.

[12] R. D. Driver, Ordinary and Delay Differential Equations, Appl. Math. Sci. 20, Springer, New York, 1977.

[13] M. Ehrnström, On radial solutions of certain semi-linear elliptic equations, Nonlinear Anal. 64 (2006), 1578-1586.

[14] - Positive solutions for second-order nonlinear differential equations, ibid., 16081620 .

[15] J. Hale, Theory of Functional Differential Equations, 2nd ed., Appl. Math. Sci. 3, Springer, New York, 1977.

[16] J. K. Hale and S. M. Verduyn Lunel, Introduction to Functional Differential Equations, Appl. Math. Sci. 99, Springer, New York, 1993.

[17] A. G. Kartsatos, Advanced Ordinary Differential Equations, 2nd ed., Mancorp, Tampa, FL, 1993.

[18] I.-G. E. Kordonis and Ch. G. Philos, On the oscillation of nonlinear two-dimensional differential systems, Proc. Amer. Math. Soc. 126 (1998), 1661-1667.

[19] T. Kusano and W. F. Trench, Existence of global solutions with prescribed asymptotic behavior for nonlinear ordinary differential equations, Ann. Mat. Pura Appl. 142 (1985), 381-392.

[20] - - - Global existence theorems for solutions of nonlinear differential equations with prescribed asymptotic behaviour, J. London Math. Soc. (2) 31 (1985), 478-486.

[21] W. T. Li and S. S. Cheng, Limiting behaviours of non-oscillatory solutions of a pair of coupled nonlinear differential equations, Proc. Edinburgh Math. Soc. 43 (2000), 457-473.

[22] W. T. Li and X. Yang, Classifications and existence criteria for positive solutions of systems of nonlinear differential equations, J. Math. Anal. Appl. 298 (2004), 446-462.

[23] Y. Liu, Existence and unboundedness of positive solutions for singular boundary value problems on half-line, Appl. Math. Comput. 144 (2003), 543-556.

[24] H. Maagli and S. Masmoudi, Existence theorem of nonlinear singular boundary value problem, Nonlinear Anal. 46 (2001), 465-473.

[25] K. G. Mavridis, Ch. G. Philos and P. Ch. Tsamatos, Existence of solutions of a boundary value problem on the half-line to second order nonlinear delay differential equations, Arch. Math. (Basel) 86 (2006), 163-175.

[26] - - - - - Multiple positive solutions for a second order delay boundary value problem on the half-line, Ann. Polon. Math. 88 (2006), 173-191.

[27] J. D. Mirzov, Asymptotic Properties of Solutions of Systems of Nonlinear Nonautonomous Ordinary Differential Equations, Folia Facultatis Scientiarum Naturalium Universitatis Masarykianae Brunensis Mathematica 14, Masaryk Univ., Brno, 2004.

[28] O. G. Mustafa and Y. V. Rogovchenko, Global existence of solutions with prescribed asymptotic behavior for second-order nonlinear differential equations, Nonlinear Anal. 51 (2002), 339-368.

[29] Ch. G. Philos, Asymptotic behaviour of a class of nonoscillatory solutions of differential equations with deviating arguments, Math. Slovaca 33 (1983), 409-428.

[30] - Positive increasing solutions on the half-line to second order nonlinear delay differential equations, Glasg. Math. J., to appear. 
[31] Ch. G. Philos, Y. G. Sficas and V. A. Staikos, Some results on the asymptotic behavior of nonoscillatory solutions of differential equations with deviating arguments, J. Austral. Math. Soc. Ser. A 32 (1982), 295-317.

[32] Ch. G. Philos and V. A. Staikos, A basic asymptotic criterion for differential equations with deviating arguments and its applications to the nonoscillation of linear ordinary equations, Nonlinear Anal. 6 (1982), 1095-1113.

[33] J. Schauder, Der Fixpunktsatz in Funktionalräumen, Studia Math. 2 (1930), 171180 .

[34] A. Tychonoff, Ein Fixpunktsatz, Math. Ann. 111 (1935), 767-776.

[35] E. Wahlén, Positive solutions of second-order differential equations, Nonlinear Anal. 58 (2004), 359-366.

[36] B. Yan, Multiple unbounded solutions of boundary value problems for second-order differential equations on the half-line, ibid. 51 (2002), 1031-1044.

[37] B. Yan and Y. Liu, Unbounded solutions of the singular boundary value problems for second order differential equations on the half-line, Appl. Math. Comput. 147 (2004), 629-644.

[38] X. Yang, Nonoscillatory solutions of nonlinear differential systems, Comput. Math. Appl. 46 (2003), 1347-1362.

[39] Z. Yin, Monotone positive solutions of second-order nonlinear differential equations, Nonlinear Anal. 54 (2003), 391-403.

[40] Z. Zhao, Positive solutions of nonlinear second order ordinary differential equations, Proc. Amer. Math. Soc. 121 (1994), 465-469.

Department of Mathematics

University of Ioannina

P.O. Box 1186

45110 Ioannina, Greece

E-mail: cphilos@cc.uoi.gr

Received 18.12.2006

and in final form 14.5.2007 